\title{
Relações entre diversidade íctia e fatores hidrodinâmicos de um riacho na bacia do rio Uruguai
}

\author{
Vander Kaufmann ${ }^{1}$ \& Adilson Pinheiro ${ }^{1,2}$ \\ ${ }^{1}$ Programa de Pós-Graduação em Engenharia Ambiental, \\ Universidade Regional de Blumenau, \\ Rua São Paulo, 3250, CEP 89030-000, Blumenau, SC, Brazil \\ ${ }^{2}$ Autor para correspondência: Adilson Pinheiro, e-mail: pinheiro@furb.br
}

KAUFMANN, V. \& PINHEIRO, A. Relationship between ichthyofaunal diversity and hydrodynamic factors in a stream of Uruguay river basin. Biota Neotrop., 9(1): http://www.biotaneotropica.org.br/v9n1/en/ abstract?article+bn00809012009.

\begin{abstract}
Physical, chemical and environmental factors in watercourses can exert influence on the structure and diversity of species of aquatic biota. Among the physical factors there are hydrodynamic conditions. Disposal variations result into complex dynamics of fish populations and communities. This study aims at assessing the correlation between hydrodynamic variables in the Fortaleza stream, affluent of Uruguay River, and species diversity. The methodology included the collection of hydrodynamic (average depth of the water column, average water flow speed and discharged water in the pluvial metric section) and biological (fish collection in the areas of influence on pluvial metric sections) data. Shannon diversity indices, equitability and the similarity index were calculated with biological data. It has been collected a sample with 527 fishes. There were 25 species, being 4 exotic ones and 4 species not-yet-classified. Such species included 4 orders, 7 families and 13 genera classified. The Shannon index ranged from 1.43 to 2.68 nats/individual. The equitability index was always above 0.8 indicating that individuals are well distributed among different species. The similarity index of Jaccard ranged between 20 and $80 \%$. The lowest figures were obtained from the sample area located near the source. The correlations between the Shannon index and hydrodynamic features resulted in $\mathrm{R}^{2}$ coefficients ranging between 0.59 and 0.83 . These results show that diversity of species is influenced by the hydrodynamic characteristics. Nevertheless, either depth of water column or discharge average speed is not significant enough to explain the spatial variability of the diversity of species.
\end{abstract}

Keywords: streamflow, fish diversity, biologic indexes.

KAUFMANN, V. \& PINHEIRO, A. Relações entre diversidade íctia e fatores hidrodinâmicos de um riacho na bacia do rio Uruguai. Biota Neotrop., 9(1): http://www.biotaneotropica.org.br/v9n1/pt/ abstract?article+bn00809012009.

Resumo: Fatores físicos, químicos e ambientais em um curso de água podem influenciar a estrutura e a diversidade das espécies da biota aquática. Entre os fatores físicos têm-se as condições hidrodinâmicas. Variações do escoamento resultam em dinâmicas complexas das populações e comunidades de peixes. Este trabalho tem por objetivo avaliar as correlações entre as variáveis hidrodinâmicas no Arroio Fortaleza, afluente do rio Uruguai, e a diversidade das espécies. A metodologia compreendeu a coleta de dados hidrodinâmicos (profundidade média da coluna de água, velocidade média do escoamento e vazão escoada na seção fluviométrica) e biológicos (coleta de peixes nas áreas de influência das seções fluviométricas). Com os dados biológicos foram calculados os seguintes índices: o de diversidade de Shannon, o de equitabilidade e o de similaridade. Coletaram-se 527 exemplares, de 25 espécies, dentre elas 4 exóticas e 4 ainda não classificadas. Essas 25 espécies estão classificadas em 4 ordens, 7 famílias e 13 gêneros. O índice de Shannon variou entre 1,43 e 2,68 nats/indivíduo. O índice de equitabilidade foi sempre superior a 0,8 , indicando que os indivíduos estão bem distribuídos entre as diferentes espécies. O índice de similaridade de Jaccard variou entre 20 e 80\%. Os menores valores foram obtidos para a área amostral situada próximo da nascente. As correlações entre o índice de Shannon e as características hidrodinâmicas resultaram em coeficientes $\mathrm{R}^{2}$ variando entre 0,59 e 0,83 . Estes resultados evidenciam que a diversidade das espécies é influenciada pelas características hidrodinâmicas. No entanto, a profundidade da coluna de água ou a velocidade média do escoamento não são suficientemente significativas para explicar a variabilidade espacial da diversidade das espécies isoladamente.

Palavras-chave: escoamento, diversidade de peixes, índices biológicos. 


\section{Introdução}

A bacia do rio Uruguai drena os estados de Santa Catarina e do Rio Grande do Sul no Brasil. As características hidrodinâmicas de seus cursos de água estão sendo modificadas com a implantação de uma série de barragens, visando à produção de hidroeletricidade. Elas são implantadas em cascatas, com transformação dos ambientes lóticos em ambientes lênticos. A avaliação dos impactos destas modificações pode ser realizada a partir das inter-relações entre as variáveis do meio físico e as que representam a biota aquática.

A diversidade de peixes de água doce no Brasil tem sido considerada como a maior de todo o mundo (Lowe-McConnell 1999, Buckup et al. 2007). No entanto, uma parcela significativa desta biota aquática ainda não tem sido adequadamente estudada e permanece insuficientemente conhecida. Assim, poucos estudos têm sido realizados buscando avaliar as interferências de componentes do meio físico sobre a diversidade das espécies encontradas nos rios brasileiros.

Os gradientes ambientais podem influenciar a distribuição e os atributos das comunidades locais. Matthews (1998) apresenta as ligações entre as comunidades de peixes e a qualidade química da água ( $\mathrm{pH}$, condutividade e teor de oxigênio), suas propriedades físicas (temperatura, transparência, características do escoamento), a disponibilidade dos habitats (reprodução, abrigo) e os recursos alimentares. Estas propriedades locais estão ligadas às características dos meios aquáticos e aos seus condicionantes ambientais, tais como clima e bacia hidrográfica.

Alguns estudos mostram que a estrutura da comunidade e a diversidade das espécies da biota aquática são afetadas por condições físicas e ambientais locais como condições hidrodinâmicas (Marchetti \& Moyle 2001, Lamouroux \& Cattanéo 2006), o substrato (Humpl \& Pivnicka 2006, Vlach et al. 2005), a vegetação ciliar (Growns et al. 2003), a composição química (Lappaleinen \& Soininen 2006) e a temperatura (Buisson et al. 2007). As condições hidrodinâmicas de um curso de água influenciam em seus ciclos de vida (Strange et al. 1993, Hilderbrand 1999, Wildhaber et al. 2000, Goldstein et al. 2007). Variações longitudinais e laterais do escoamento resultam em complexas dinâmicas espaciais e temporais das populações e comunidades de peixes, particularmente porque fluxos altos e baixos influenciam a sobrevivência (Schlosser 1991). De acordo com Brown \& Matthews (1995), a diversidade tende a diminuir em direção às cabeceiras dos riachos. Isto sugere a existência de relação entre as características do hábitat e a riqueza de espécies.

Neste sentido, este trabalho tem por objetivo avaliar as correlações entre as variáveis hidrodinâmicas de um rio de pequena ordem de acordo com ordenamento proposto pelo método de Horton (Silveira, 2004) e a diversidade das espécies. O curso de água é afluente do rio Uruguai, no seu trecho médio e se situa na região extrema oeste do estado de Santa Catarina.

\section{Materiais e Metodos}

A metodologia compreende a coleta de dados hidrodinâmicos e biológicos e, o tratamento e análise desses dados. Os dados hidrodinâmicos abrangem a profundidade média da coluna de água, a velocidade média do escoamento e a vazão escoada na seção fluviométrica. Os dados biológicos incluem os peixes coletados nas áreas de influência das seções fluviométricas, denominadas áreas amostrais.

Este estudo foi realizado no Arroio Fortaleza, que é um tributário do rio Uruguai no município de Itapiranga - SC. Sua nascente localiza-se no município de São João do Oeste, na cota 537 m e o exutório encontra-se na cota $162 \mathrm{~m}$ acima do nível do mar. A bacia do Arroio Fortaleza localiza-se entre as coordenadas $27^{\circ} 03^{\prime} 796^{\prime \prime} \mathrm{S}$ e $53^{\circ} 57^{\prime} 851^{\prime \prime} \mathrm{W} ; 27^{\circ} 17^{\prime} 600^{\prime \prime} \mathrm{S}$ e $53^{\circ} 64^{\prime} 597^{\prime}$ 'W, drenando uma área de $64,28 \mathrm{~km}^{2}$ (Figura 1). Foram selecionadas sete áreas amostrais para desenvolvimento deste estudo, descritas a seguir.

- Área 1: situada a $2 \mathrm{~km}$ da nascente. É o primeiro local onde se encontram peixes no Arroio Fortaleza. É constituída de uma queda de água com aproximadamente $13 \mathrm{~m}$ de altura, com profundidade de, no máximo, 1,3 m. Suas margens são íngremes com boa parte de paredão rochoso, com algumas plantas arbóreas ralas. Não se registram plantas oleaginosas e frutíferas. O leito do rio nesse local é rochoso com sedimentação de terra em sua margem esquerda, com depósito de restos vegetais trazidos pelas enxurradas.

- Área 2: situa-se a cerca de $3 \mathrm{~km}$ da nascente, com três quedas de água consecutivas. Em sua borda lateral e de saída apresenta sedimentos e pedras. Uma grande coluna rochosa se ergue ao redor do córrego. A margem apresenta plantas com sementes oleaginosas, plantas frutíferas e também uma área com plantação de araucárias Araucaria angustifolia (Bertol.) O. Kuntze. Há ocorrência de túneis submersos e a presença de nascente de água.

- Área 3: localiza-se a 20 minutos da cidade (a montante do perímetro urbano), na represa superior de captação de água para abastecimento público (três represas para captação de água existem em seqüência). As águas lênticas formadas pelo lago têm uma extensão de $350 \mathrm{~m}$. O fundo é caracterizado por sedimentos orgânicos do solo trazido pelas fortes chuvas. A margem ciliar do lago apresenta pastagens para o gado, com poucas árvores, geralmente frutíferas nativas. No terço superior do lago ocorre uma vegetação mais diversificada com entrada de um pequeno tributário.

- Área 4: situada a cerca de $3 \mathrm{~km}$ do centro da cidade (jusante). Na margem do local ocorrem plantas de médio porte bem diversificadas. Toda borda apresenta rocha com pequena presença de sedimento e restos orgânicos. Existe uma pequena entrada de água sulfurada, proveniente do Parque de Águas Termais construído no ano de 2001. O parque mantém uma boa área ciliar bem conservada. O solo tem declínio acentuado, vegetação com características predominantes de leguminosa e frutífera, presença de Arundinaria sp. densas (planta aparentada com o bambu). A mata tem porte elevado, cobrindo praticamente todo

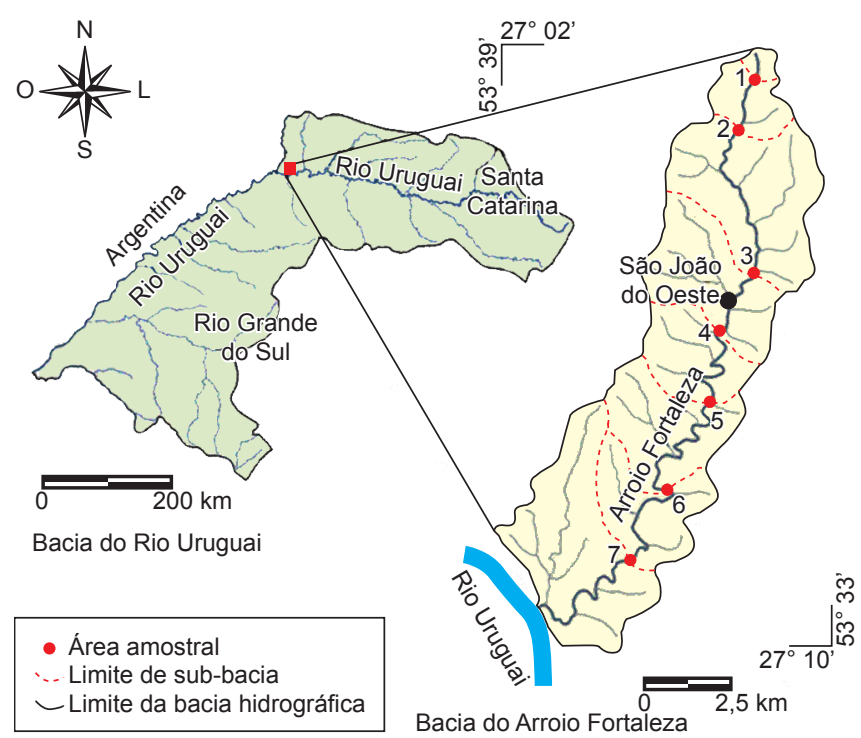

Figura 1. Bacia do Arroio Fortaleza com localização das áreas amostrais.

Figure 1. Fortaleza river basin with location of sampling areas. 
o leito do rio. Não há presença de cultivo agrícola próximo às margens.

- Área 5: situada a cerca de $5 \mathrm{~km}$ à jusante da cidade, apresenta área nativa próxima à margem direita superior do córrego. A vegetação é fechada permitindo pouca insolação ao rio. As árvores das bordas são constituídas de arbustos de porte elevado. Encontram-se áreas cultivadas próximas do leito e entrada de bovinos. Uma de suas margens é formada por um paredão rochoso, com presença de fendas submersas. O leito do rio é rochoso com sedimentos grosseiros no terço final. No verão ocorrem acampamentos periódicos e banhos, pois as águas são cristalinas nesta época.

- Área 6: localizada em um pequeno vilarejo com o nome de Fortaleza de onde surgiu o nome do rio. Neste trecho do rio as margens encontram-se devastadas, com sedimentação de material particulado, transportado pelos afluentes e pelo rio. A vegetação de entorno é pouca, acarretando a maior insolação do rio quando comparada a todas as áreas amostradas. As plantas mais freqüentes são as gramíneas.

- Área 7: o rio caracteriza-se por uma grande sinuosidade. O local apresenta extensão entre as corredeiras de aproximadamente $300 \mathrm{~m}$ com bom volume de água. Não existe presença de mata ciliar, apenas pequenos arbustos e pastagem perene. Em sua parte superior o rio tem margens de terra com deposição de sedimentos. No quarto inferior é formado por depósito de sedimentos e fundo pedregoso.

O clima da bacia, segundo a classificação de Köeppen (Ayoade, 1996), é Cfa, subtropical úmido, sem estação seca, com verão quente. A temperatura média anual varia de 17,9 a $19,8^{\circ} \mathrm{C}$. A precipitação média anual é da ordem de $1.800 \mathrm{~mm}$, sendo bem distribuída nas quatro estações do ano. A insolação varia de 2.117 a 2.395 horas. A geologia da área é constituída basicamente por rochas basálticas oriundas do derrame basáltico. Os rios possuem cursos sinuosos e vales encaixados, com patamares nas vertentes. A vegetação original é de floresta estacional decidual. Atualmente, é ocupada com agricultura de culturas cíclicas (EPAGRI, 1999). Os solos predominantes na bacia são cambissolos e neossolos, caracterizados pela alta fertilidade natural, pedregosidade freqüente e profundidade média pequena. $\mathrm{O}$ relevo predominante é fortemente ondulado. O leito do rio é formado basicamente pela rocha basáltica, com pequenos locais com sedimentos e muita presença de pedras.

A seção fluviométrica é dividida em verticais, nos quais é determinada a profundidade da coluna de água e a velocidade do escoamento. As velocidades foram determinadas a $30 \%$ do fundo do leito e $30 \%$ da superfície da lâmina de água. A velocidade do escoamento foi medida com o Molinete C-20 e Contador Z-30, da marca OTT. O molinete consiste de uma hélice presa a uma haste e um "contagiros", que mede a velocidade da água que passa pela hélice. Assim, quando posicionado em determinada profundidade, ele determina a velocidade do ponto.

A velocidade é determinada através da expressão $V=a . n+b$, onde a e b são parâmetros do aparelho, calibrados em laboratório. O molinete empregado permite a medição da velocidade em cursos de água com profundidades inferiores a $1,90 \mathrm{~m}$. O produto da velocidade pela área fornece a vazão escoada na seção transversal.

As áreas amostrais foram definidas através de expedições e investigações, nas quais foram consultados os moradores ribeirinhos que relataram a existência de determinadas espécies. Os quesitos básicos para a determinação do local de coleta foram: a presença de túneis submersos (tocas), a presença de grandes pedras no leito e mata ripária pobre. As coletas foram realizadas no período de janeiro a maio de 2003, compreendendo campanhas de amostragem de 1 a 3 dias sucessivos.
A coleta dos exemplares foi efetuada com materiais de pesca convencionais (rede, fisga, anzóis, esperas, cevas), em diferentes dias e turnos com condições climáticas variadas. Após a captura os exemplares foram fixados com álcool a $70 \%$ e formol 10\%, numerados por posto de coleta e levados à Universidade de Palmas no Paraná. Ali foram analisados, fotografados, classificados e depois repassados ao museu de Guarapuava - PR.

$\mathrm{Na}$ identificação, os exemplares de peixes coletados foram comparados com o Catálogo Ilustrado de Peixes do Alto Rio Uruguai (Zaniboni Filho, 2004), catálogo de peixes da bacia do Rio Iguaçu (Severi \& Cordeiro 1994) e do Fischbase. Este último é um software produzido especificamente para taxar as espécies animais, criado pela Comunidade Internacional de Ictiologia.

Para uma melhor compreensão dos dados sobre a distribuição espacial dos peixes coletados, foram calculados os índices de diversidade de Shannon, de equitabilidade e de similaridade. O índice de Shannon é expresso por:

$$
\mathrm{H}=-\sum_{\mathrm{i}=1}^{\mathrm{N}} \mathrm{p}_{\mathrm{i}} \cdot \operatorname{Ln}\left(\mathrm{p}_{\mathrm{i}}\right)
$$

Sendo $\mathrm{p}_{\mathrm{i}}=\frac{\mathrm{n}_{\mathrm{i}}}{\mathrm{N}}$, onde $\mathrm{H}$ é a diversidade, ni é o número de indivíduos do taxa i e $\mathrm{N}$ é o número total de indivíduos da comunidade.

O índice de equitabilidade representa a distribuição dos indivíduos em seu habitat. Este índice é expresso por:

$$
\mathrm{E}=\frac{\mathrm{H}}{\mathrm{H}_{\max }}
$$

sendo $\mathrm{H}_{\max }=\mathrm{h} \mathrm{S}$, onde $\mathrm{S}$ é o número de espécies da comunidade.

A similaridade entre as áreas amostrais foi estimada através do índice de Jaccard (J) expresso por

$$
J=\frac{a}{a+b+c}
$$

onde a é o número de espécies comuns aos pontos $\mathrm{A}$ e $\mathrm{B}$ a serem comparados; b é o número de espécies que ocorrem no ponto $\mathrm{A}$, e não ocorrem no ponto $\mathrm{B}$; c é o número de espécies que ocorrem no ponto $\mathrm{B}$, e não ocorrem no ponto $\mathrm{A}$.

\section{Resultados}

Foram realizadas 17 campanhas de coleta. Na Tabela 1 são apresentadas as distribuições e o total dos exemplares coletados em cada área amostral, tendo sido coletados 527 exemplares. A quantidade de exemplares coletados foi variável entre as áreas amostrais, sendo o menor número o da área 1, com 47, e o maior, o da área 6, com 100. A área 1 situa-se mais a montante, próxima da nascente. O Astyanax bimaculatus (Linnaeus, 1758) e o Gymnogeopagus gymnogenys (Hensel, 1870) foram coletados em todas as áreas e o primeiro em maior quantidade, representando cerca de $18 \%$ dos exemplares totais.

Em termos de espécies, foram coletadas, ao todo, 25, sendo 4 exóticas e 4 ainda não classificadas. Estas espécies foram classificadas em 4 ordens, 7 famílias e 13 gêneros. Não foram identificadas as famílias de 2 exemplares. O gênero mais abundante foi o Astyanax spp., com um total de 168 exemplares, representando cerca de $32 \%$ do total. A família Characidae representou cerca de $39 \%$ dos exemplares coletados. Pessano et al. (2005) encontraram 37 espécies, 27 gêneros e 14 famílias no arroio Quarai-Chico, afluente do rio Uruguai, situado em área de conservação ambiental.

A Tabela 2 apresenta os índices de diversidade de Shannon e de equitabilidade para cada área amostral. O índice de Shannon variou entre 1,43 e 2,68 nats/indivíduo. O menor valor foi determinado na área amostral 1 e o maior, na 6 . Observa-se que o valor médio deste 
Tabela 1. Distribuição de indivíduos coletados em famílias e espécies nas áreas amostrais na bacia do Arroio Fortaleza.

Table 1. Distribution of collected individuals from the sampling areas at the Fortaleza river basin.

\begin{tabular}{|c|c|c|c|c|c|c|c|c|}
\hline \multirow[t]{2}{*}{ Espécies } & \multicolumn{7}{|c|}{ Áreas de coleta } & \multirow[b]{2}{*}{$\mathbf{N}$} \\
\hline & 1 & 2 & 3 & 4 & 5 & 6 & 7 & \\
\hline \multicolumn{9}{|l|}{ CHARACIDAE } \\
\hline Astyanax fasciatus (Cuvier, 1819) & 0 & 0 & 30 & 0 & 2 & 5 & 1 & 38 \\
\hline Astyanax bimaculatus (Linnaeus, 1758) & 12 & 16 & 20 & 6 & 20 & 15 & 5 & 94 \\
\hline Astyanax gr. Scabripinnis (Jenyns, 1842) & 0 & 0 & 7 & 4 & 7 & 7 & 11 & 36 \\
\hline Oligosarcus brevioris Menezes, 1987 & 0 & 3 & 5 & 5 & 9 & 4 & 0 & 26 \\
\hline \multirow[t]{2}{*}{ Oligosarcus jenynsii (Günther, 1864) } & 0 & 0 & 0 & 0 & 1 & 2 & 7 & 10 \\
\hline & & & & & & & Total & 204 \\
\hline \multicolumn{9}{|l|}{ CURIMATIDAE } \\
\hline Steindachnerina brevipinna (Eigenmann \& Eigenmann, 1889) & 0 & 0 & 0 & 0 & 16 & 0 & 25 & 41 \\
\hline \multicolumn{9}{|l|}{ ERYTHRINIDAE } \\
\hline Hoplias lacerdae (Miranda-Ribeiro, 1908) & 0 & 4 & 3 & 0 & 3 & 2 & 1 & 13 \\
\hline \multirow[t]{2}{*}{ Hoplias malabaricus (Bloch, 1794) } & 0 & 2 & 2 & 1 & 3 & 3 & 3 & 14 \\
\hline & & & & & & & Total & 27 \\
\hline \multicolumn{9}{|l|}{ CYPRINIDAE } \\
\hline Ctenopharingodon idellus (Valenciennes, 1850) & 0 & 0 & 0 & 0 & 0 & 0 & 1 & 1 \\
\hline Cyprinus carpio (Linnaeus, 1758) & 0 & 0 & 0 & 0 & 0 & 2 & 1 & 3 \\
\hline \multirow[t]{2}{*}{ Cyprinus carpio (Linnaeus, 1758) } & 0 & 0 & 0 & 1 & 0 & 2 & 1 & 4 \\
\hline & & & & & & & Total & 8 \\
\hline
\end{tabular}

\section{CICHLIDAE}

Geophagus brasilliensis (Quoy \& Gaimard, 1824)

Gymnogeopagus gymnogenys (Hensel, 1870)

Oreochromis niloticus (Linnaeus, 1758)

Crenicichla missioneira (Lucena \& Kullander), 1992

Crenicichla minuano Lucena \& Kullander, 1992

Cichlasoma aff. facetum (Jenyns, 1842)

Não identificado

\begin{tabular}{rrrrrrrr}
0 & 2 & 2 & 2 & 3 & 12 & 1 & 22 \\
22 & 7 & 5 & 5 & 2 & 5 & 4 & 50 \\
3 & 2 & 1 & 0 & 0 & 1 & 0 & 7 \\
0 & 0 & 0 & 0 & 2 & 3 & 0 & 5 \\
0 & 2 & 3 & 7 & 3 & 11 & 6 & 32 \\
0 & 0 & 0 & 5 & 0 & 0 & 0 & 5 \\
0 & 0 & 0 & 2 & 2 & 0 & 0 & 4 \\
& & & & & & Total & 125 \\
\hline
\end{tabular}

\section{LORICARIIDAE}

Hypostomus commersonii (Valenciennes, 1836)

Rineloricaria $\mathrm{sp.}$

$\begin{array}{llllllll}0 & 12 & 0 & 3 & 5 & 11 & 3 & 34\end{array}$

Não identificado

$\begin{array}{rrrrrrrrr}0 & 0 & 0 & 0 & 1 & 0 & 0 & 1\end{array}$

\begin{tabular}{|c|c|c|c|c|c|c|c|c|}
\hline & & & & & & & Total & 56 \\
\hline \multicolumn{9}{|l|}{ HEPTAPTERIDAE } \\
\hline Rhamdia quelen (Quoy \& Gaimard, 1824) & 2 & 2 & 2 & 0 & 1 & 3 & 3 & 13 \\
\hline \multirow[t]{2}{*}{ Rhamdia sp. } & 5 & 5 & 4 & 2 & 4 & 7 & 4 & 31 \\
\hline & & & & & & & Total & 44 \\
\hline \multicolumn{9}{|l|}{ Não identificado } \\
\hline Não identificado (Farelo) & 0 & 5 & 7 & 4 & 2 & 2 & 1 & 21 \\
\hline \multirow[t]{2}{*}{ Não identificado (Espada) } & 0 & 0 & 0 & 0 & 0 & 0 & 1 & 1 \\
\hline & & & & & & & Total & 22 \\
\hline Total & 47 & 67 & 98 & 48 & 88 & 100 & 79 & 527 \\
\hline
\end{tabular}

índice, entre as áreas, é igual a 2,27 $\pm 0,41$ nats/indivíduo. Estes valores representam grande diversidade de espécies. $\mathrm{O}$ índice de equitabilidade foi sempre superior a 0,8 , indicando que os indivíduos estão bem distribuídos entre as diferentes espécies. Valores elevados destes índices são indicativos de ambientes complexos, com múltiplas relações, significando um ecossistema com boa estabilidade, apesar de o uso da terra ser intenso (Tischer \& Ferrão Santos, 2001).
A Tabela 3 apresenta os índices de similaridade entre as áreas amostrais. A área 1 apresenta baixa similaridade com as demais áreas e o índice de similaridade de Jaccard variou entre 20 e 46\%. Entre as outras áreas verifica-se que existe similaridade mais elevada, no entanto, o valor máximo foi de $80 \%$, podendo indicar que o curso de água não seja, talvez, totalmente homogêneo, sobretudo em relação à área 4 em que ocorre uma redução dos índices de similaridade. Neste 
Tabela 2. Índices de Shannon e Equitabilidade nas áreas amostrais na bacia do Arroio Fortaleza.

Table 2. Shannon and equitability indexes in the sampling areas at the Fortaleza river basin.

\begin{tabular}{ccccc}
\hline Área & $\mathbf{N}$ & $\mathbf{H}$ (nats/ind) & $\mathbf{H}_{\text {máx }}$ & $\mathbf{E}$ \\
\hline 1 & 47 & 1,43 & 1,79 & 0,80 \\
2 & 67 & 2,30 & 2,56 & 0,90 \\
3 & 98 & 2,25 & 2,71 & 0,83 \\
4 & 48 & 2,47 & 2,64 & 0,94 \\
5 & 88 & 2,51 & 2,94 & 0,85 \\
6 & 100 & 2,68 & 2,94 & 0,91 \\
7 & 79 & 2,34 & 2,89 & 0,81 \\
Média & $75 \pm 22$ & $2,27 \pm 0,41$ & $2,64 \pm 0,40$ & $0,86 \pm 0,06$ \\
\hline
\end{tabular}
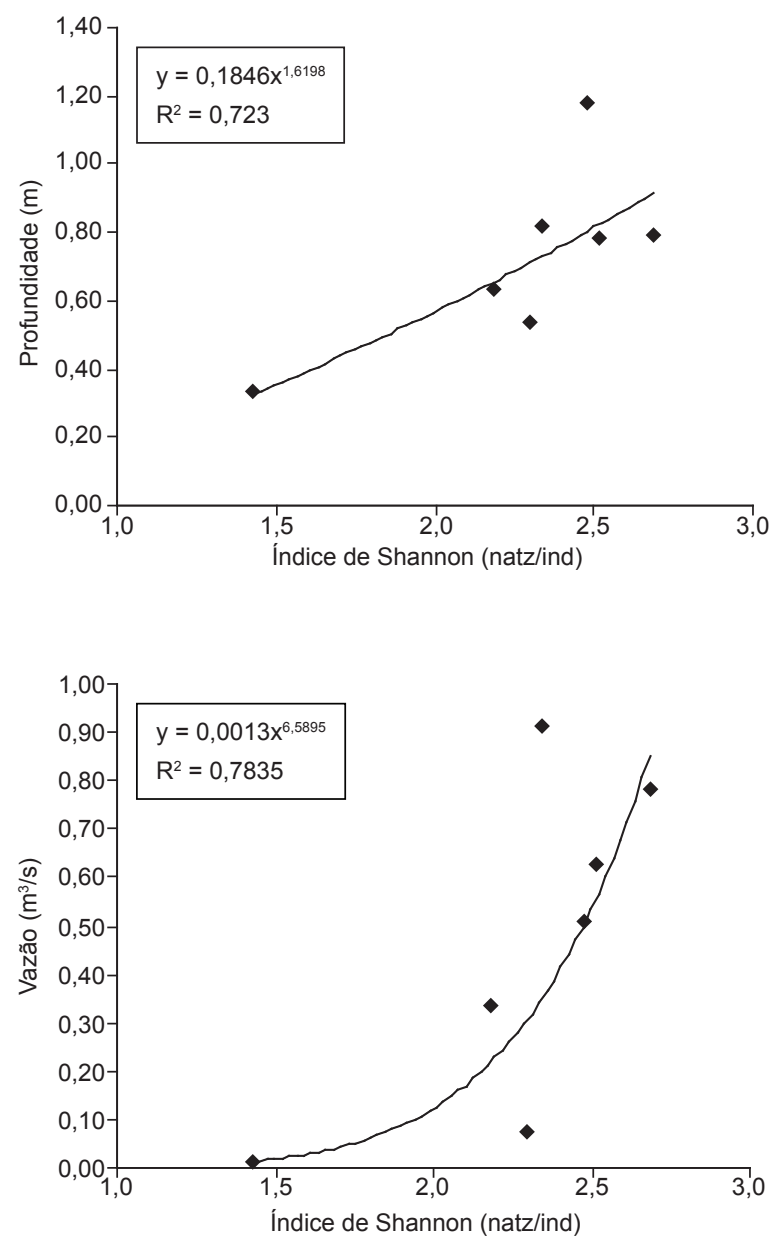

Tabela 3. Índices de similaridade de Jaccard (\%) entre as áreas amostrais na bacia do Arroio Fortaleza.

Table 3. Jaccard similarity indexes among sampling areas at the Fortaleza river basin.

\begin{tabular}{crrrrrrc}
\hline Áreas & $\mathbf{1}$ & $\mathbf{2}$ & $\mathbf{3}$ & $\mathbf{4}$ & $\mathbf{5}$ & $\mathbf{6}$ & $\mathbf{7}$ \\
\hline 1 & 100 & - & - & - & - & - & - \\
2 & 46 & 100 & - & - & - & - & - \\
3 & 43 & 80 & 100 & - & - & - & - \\
4 & 25 & 38 & 56 & 100 & - & - & - \\
5 & 25 & 60 & 65 & 57 & 100 & - & - \\
6 & 32 & 68 & 74 & 57 & 65 & 100 & - \\
7 & 20 & 48 & 52 & 45 & 61 & 68 & 100 \\
\hline
\end{tabular}
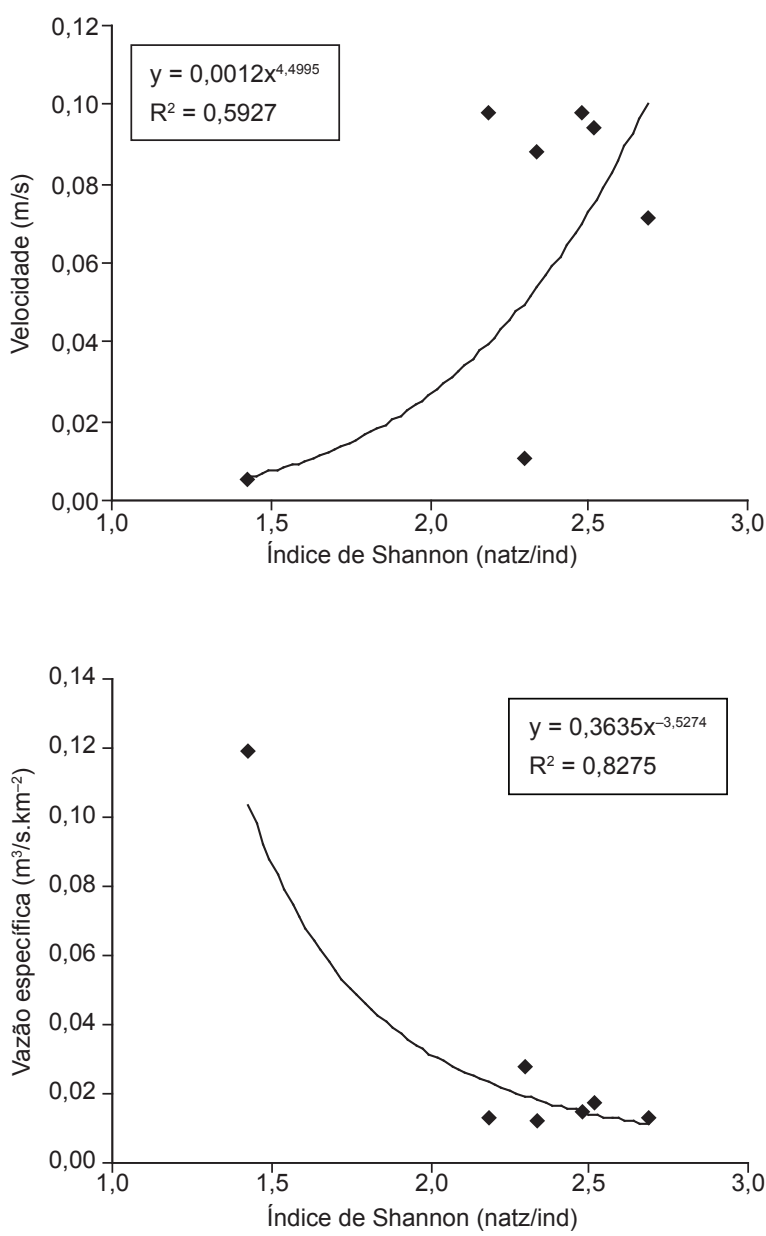

Figura 2. Correlação entre os índices de Shannon e as variáveis hidrodinâmicas (profundidade, velocidade, vazão e vazão específica do escoamento) na bacia do Arroio Fortaleza.

Figure 2. Correlation between Shannon indexes and hydrodynamic variables (depth, velocity, discharge and specific discharge of flow) in the Fortaleza river basin.

ponto foram coletadas 12 espécies entre os 48 exemplares capturados. O número de exemplares capturados foi similar ao da área 1, contudo, o índice de similaridade entre estas duas áreas é baixo.

As características hidrodinâmicas medidas nas seções fluviométricas das áreas amostrais são apresentadas na Tabela 4. Verifica-se que a área amostral 4 apresentou a maior profundidade média da coluna de água, com valor de $1,178 \mathrm{~m}$. Em relação a velocidade média, as áreas amostrais 3 e 4 apresentaram valores de mesma ordem de grandeza. Isto indica que a evolução espacial das profundidades da coluna de água e da velocidade média nas seções transversais é 
Tabela 4. Áreas de drenagem e características hidrodinâmicas (profundidade e velocidades) dos escoamentos nas áreas amostrais na bacia do Arroio Fortaleza.

Table 4. Drainage surface and hydrodynamic characteristics (depth and velocity) of flows in the sampling areas at the Fortaleza river basin.

\begin{tabular}{cccc}
\hline Área & $\begin{array}{c}\text { Área de } \\
\text { drenagem }\left(\mathbf{k m}^{2}\right)\end{array}$ & $\begin{array}{c}\text { Profundidade } \\
(\mathbf{m})\end{array}$ & $\begin{array}{c}\text { Velocidade } \\
(\mathbf{m} / \mathbf{s})\end{array}$ \\
\hline 1 & 0,71 & $0,333 \pm 0,043$ & $0,005 \pm 0,002$ \\
2 & 3,99 & $0,538 \pm 0,038$ & $0,011 \pm 0,003$ \\
3 & 17,90 & $0,630 \pm 0,288$ & $0,098 \pm 0,055$ \\
4 & 27,36 & $1,178 \pm 0,126$ & $0,098 \pm 0,030$ \\
5 & 33,52 & $0,781 \pm 0,125$ & $0,094 \pm 0,021$ \\
6 & 42,04 & $0,793 \pm 0,071$ & $0,071 \pm 0,024$ \\
7 & 48,89 & $0,819 \pm 0,129$ & $0,088 \pm 0,038$ \\
\hline
\end{tabular}

Índices de Shannon e Equitabilidade nas áreas amostrais na bacia do Arroio Fortaleza.

variável, podendo afetar a similaridade e diversidade dos peixes no ecossistema aquático.

\section{Discussões}

O efeito das características hidrodinâmicas sobre a similaridade e a diversidade das espécies é verificado através da correlação entre o índice de Shannon e a profundidade da coluna de água, a velocidade média do escoamento, vazão e vazão específica. As correlações e os coeficientes de determinação obtidos são apresentados na Figura 2. Os coeficientes $R^{2}$ variaram entre 0,59 e 0,83 . O maior valor foi obtido para a vazão específica, a qual representa a vazão gerada por unidade da área de drenagem da bacia. Este valor está diretamente relacionado com o tipo e estrutura do solo e o uso da terra (Dormann et al. 2007, Snelder 2004). A vazão escoada apresentou o segundo maior valor, indicando que a diversidade das espécies é explicada pela quantidade de água escoada. A profundidade da coluna de água e a velocidade também explicam de forma significativa a diversidade das espécies.

A vazão escoada está relacionada com a profundidade da coluna de água e com a velocidade da corrente líquida. A vazão específica, por sua vez, integra, ainda. a área de drenagem até a seção fluviométrica considerada. Estes resultados evidenciam que a diversidade das espécies é influenciada por um conjunto de fatores, tais como os hidrodinâmicos. A profundidade da coluna de água ou a velocidade média do escoamento não são suficientemente significativas para explicar a variabilidade espacial da diversidade das espécies isoladamente. Vieira e Shibatta (2007) encontraram valores similares para a correlação da riqueza de espécies de peixes com a velocidade do escoamento em um riacho urbano alterado. Quando estes fatores são associados entre si, resultando na vazão escoada, a qual permite explicar mais fortemente a variação espacial da diversidade de espécies. Por outro lado, a associação deste fator com a área de drenagem da bacia contribuinte apresenta resultados ainda melhores (Pont et al. 2005). Neste caso, a área de drenagem incorpora as relações do ecossistema aquático com o meio externo, representado pelas vertentes da bacia, no qual existem processos ambientais que proporcionam trocas de matérias e energias.

É interessante notar que, geralmente, as correlações entre as condições hidrodinâmicas têm sido realizadas para determinadas espécies de peixes, principalmente em relação àquelas que são mais sensíveis ou que apresentam maior importância ecológica (Buisson et al. 2007, Lamouroux \& Jowett 2005, Vlach et al. 2005, Lamouroux \&
Capra 2002). As análises são realizadas para valores característicos de vazões tais como Q50 (vazão com frequiência de ocorrência de $50 \%$ ) e Q95 (vazão com freqüência de ocorrência de 95\%) (Bragg et al. 2005). Nos estudos de aproveitamento dos recursos hídricos para geração hidroelétrica, aparece um conflito entre as vazões remanescentes em determinados trechos dos cursos de água e a capacidade produtiva da infra-estrutura implantada. Desta forma, frente às inúmeras formas de possíveis alterações estruturais em corpos de água, torna-se necessário garantir uma vazão ecológica que possa assegurar a preservação do equilíbrio natural e a sustentabilidade dos ecossistemas aquáticos, visando à manutenção da biodiversidade (Hauer \& Lamberti 1996).

\section{Referências}

AYOADE, J.O. 1996. Introdução à climatologia para os trópicos. Editora Bertrand Brasil, Rio de Janeiro.

BRAGG, O.M., BLACK, A.R., DUCK, R.W. \& ROWAN, J.S. 2005. Approaching the physical-biological interface in rivers: a review of methods for ecological evaluation of flow regimes. Progress in Physical Geograph. 29(4):506-531.

BROWN, A.V. \& MATTHEWS, W.J. 1995. Streams ecosystems of the central United States. In River and stream ecosystems of the world (C.E. Cushing, K.W. Cummins \& G.W. Minshall, eds.). Elsevier, Amsterdam, p. 89-116.

BUCKUP, P.A., MENEZES, N.A. \& GUAZZI, M.S. 2007. Catálogo das espécies de peixes de água doce do Brasil. Museu Nacional, Rio de Janeiro.

BUISSON, L., BLANC, L. \& GRENOUILLET, G. 2007. Modelling stream fish species distribution in a river network: the relative effects of temperature versus physical factors. Ecol. freshw. fish. 17(2):244-257.

DORMANN, C.F., McPHERSON, J.M., ARAÚJO, M.B., BIVAND, R., BOLLIGER, J., CARL, G., DAVIES, R.G., HIRZEL, A., JETZ, W., KISSLING, W.D., KÜHN, I., OHLEMÜLLER, R., PERES-NETO, P.R., REINEKING, B., SCHRÖDER, B., SCHURR, F. M. \& WILSON, R. 2007. Methods to account for spatial autocorrelation in the analysis of species distributional data: a review. Ecography, 30(5):609-628.

EMPRESA DE PESQUISA AGROPECUÁRIA E EXTENSÃO RURAL DE SANTA CATARINA. 1999. Zoneamento agroecológico e socioeconômico do Estado de Santa Catarina. EPAGRI, Florianópolis.

GOLDSTEIN, R.M., MEADOR, M.R. \& RUHL, K.E. 2007. Relative influence of streamflows in assessing temporal variability in stream habitat. J. Am. Water Resour. Assoc. 43(3):642-650.

GROWNS, I., GEHRKE, P.C., ASTLES, K.L. \& POLLARD, D.A. 2003. A comparison of fish assemblages associated with different riparian vegetation types in the Hawkesbury-Nepean River system. Fish. manag. ecol. 10(4):209-220.

HAUER, F.R. \& LAMBERTI, G.A. 1996. Methods in stream ecology. Academic. Press, San Diego.

HILDERBRAND, R.H. 1999. Habitat sequencing and the importance of discharge in inferences. N Am J Fish Manag. 19(1):198-202.

HUMPL, M. \& PIVNICKA, K. 2006. Fish assemblages as influenced by environmental factors in streams in protected areas of the Czech Republic. Ecol. freshw. fish. 15(1):96-103.

LAMOUROUX, N., CAPRA, H. 2002. Simple predictions of instream habitat model outputs for target fish populations. Freshw. Biol. 47(8):1543-1556.

LAMOUROUX, N. \& JOWETT, I.G. 2005. Generalized instream habitat models. Can. J. Fish. Aquat. Sci. 62(1):7-14.

LAMOUROUX, N. \& CATTANÉO, F. 2006. Fish assemblages and stream hydraulics: consistent relations across spatial scales and regions. River res. appl. 22(7):727-737.

LAPPALEINEN, J. \& SOININEN, J. 2006. Latitudinal gradients in niche breadth and position - regional patterns in freshwater fish. Naturwissenschaften, 93(5):246-250. 
LOWE-McCONNELL, R.H. 1999. Estudos ecológicos de comunidades de peixes tropicais. Edusp, São Paulo.

MARCHETTI, M.P. \& MOYLE, P.B. 2001. Effects of flow regime on fish assemblages in a regulated California stream. Ecol. appl. 11(2):530-539.

MATTHEWS, W. J. 1998. Patterns in freshwater fish ecology. Chapman and Hall, New York; Norwell.

PONT, D., HUGUENY, B. \& OBERDORFF, T. 2005. Modelling habitat requirement of European fishes: do species have similar responses to local and regional constraints?. Can. J. Fish. Aquat. Sci. 62(1):163-173.

PESSANO, E.F.C., AZEVEDO, C.L.O., QUEROL, M.V.M., QUEROL, E., BRASIL, L.G., CASTRO, L.R.B., PINTO, T.B. \& CORRÊA, F.V. 2005. Ictiofauna do arroio Quarai-Chico, bacia do médio rio Uruguai, no interior do Parque Estadual do Espinilho, Rio Grande do Sul, Brasil. Biotemas, 18(2):143-153.

SCHLOSSER, I.J. 1991. Stream fish ecology: a landscape perspective. BioScience, 41(10):704-707.

SNELDER, T.H., CATTANÉO, F., SUREN, A.M. \& BIGGS, B.J.F. 2004. Is the river environment classification an improved landscape-scale classification of rivers?. J. N. Am. Benthol. Soc. 23(3):580-598.

SEVERI, W. \& CORDEIRO, A.M. 1994. Catálogo de peixes da bacia do rio Iguaçu. $1^{a}$ ed. IAP; GTZ, Curitiba.

SILVEIRA, A.L.L. 2004. Ciclo hidrológico e bacia hidrográfica. In Hidrologia, ciência e aplicação (C.E.M. Tucci, ed.). ABRH; UFRGS, Porto Alegre, p. 25-51.
STRANGE, E.M., MOYLE, P.B. \& FOIN, T.C. 1993. Interactions between stochastic and deterministic processes in stream fish community assembly. Environ. biol. fisches. 36(1):1-15.

TISCHER, M. \& FERRÃO SANTOS, M.C. 2001. Algumas considerações sobre a ictiofauna acompanhante da pesca de camarões na foz do rio São Francisco (Alagoas/Sergipe - Brasil). Bol. Técn. Cient. CEPENE. 9(1):155-165.

VIEIRA, D.B. \& SHIBATTA O.A. 2007. Peixes como indicadores da qualidade ambiental do ribeirão Esperança, município de Londrina, Paraná, Brasil. Biota neotrop. 7(1):57-65.

VLACH, P., DUŠEK, J., ŠVÁTORA, M. \& MORAVEC, P. 2005. Fish assemblage structure, habitat and microhabitat preference of five fish species in a small stream. Folia Zool. 54(4):421-431.

WILDHABER, M.L., ALLERT, A.L., SCHMITT, C.J., TABOR, V.M., MULHERN, D., POWELL, K.L. \& SOWA, S.P. 2000. Natural and anthropogenic influences on the distribution of the threatened Neosho Madtom in a Midwestern Warmwater Stream. Trans Am Fish Soc. 129(1):243-261.

ZANIBONI FILHO, E., MEURER, S., SHIBATTA, O.A. \& NUÑER, A.P.O. 2004. Catálogo de peixes do alto rio Uruguai. UFSC; Tractebel Energia, Florianópolis.

Recebido em 21/08/08 Versão Reformulada recebida em 18/11/08 Publicado em 14/01/09 
